\title{
BMJ Open Lifestyle InterVention IN Gestational diabetes (LIVING) in India, Bangladesh and Sri Lanka: protocol for process evaluation of a randomised controlled trial
}

\author{
Janani Shanthosh (D) , ${ }^{1}$ Deksha Kapoor, ${ }^{2}$ Lakshmi K Josyula, ${ }^{3,4,5}$ Anushka Patel, ${ }^{1}$ \\ Yashdeep Gupta, ${ }^{2}$ Nikhil Tandon, ${ }^{2}$ Stephen Jan, ${ }^{1}$ Helena J Teede, ${ }^{6}$ Ankush Desai, ${ }^{7}$ \\ Rohina Joshi (D) ," Devarsetty Praveen ${ }^{3,4,5}$
}

To cite: Shanthosh J, Kapoor D, Josyula LK, et al. Lifestyle InterVention IN Gestational diabetes (LIVING) in India, Bangladesh and Sri Lanka: protocol for process evaluation of a randomised controlled trial. BMJ Open 2020;10:e037774. doi:10.1136/ bmjopen-2020-037774

- Prepublication history and supplemental material for this paper are available online. To view these files, please visit the journal online (http://dx.doi. org/10.1136/bmjopen-2020037774).

Received 15 February 2020 Revised 31 July 2020 Accepted 23 September 2020

D Check for updates

(c) Author(s) (or their employer(s)) 2020. Re-use permitted under CC BY-NC. No commercial re-use. See rights and permissions. Published by BMJ.

For numbered affiliations see end of article.

Correspondence to Janani Shanthosh; jshanthosh@georgeinstitute. org.au

\section{ABSTRACT}

Introduction The development of type 2 diabetes mellitus disproportionately affects South Asian women with prior gestational diabetes mellitus (GDM). The Lifestyle InterVention IN Gestational diabetes (LIVING) Study is a randomised controlled trial of a low-intensity lifestyle modification programme tailored to women with previous GDM, in India, Bangladesh and Sri Lanka, aimed at preventing diabetes/pre-diabetes. The aim of this process evaluation is to understand what worked, and why, during the LIVING intervention implementation, and to provide additional data that will assist in the interpretation of the LIVING Study results. The findings will also inform future scale-up efforts if the intervention is found to be effective.

Methods and analysis The Reach Effectiveness Adoption Implementation Maintenance (RE-AIM) methodological approach informed the evaluation framework. Michie's Behaviour Change Theory and Normalisation Process Theory were used to guide the design of our qualitative evaluation tools within the overall RE-AIM evaluation framework. Mixed methods including qualitative interviews, focus groups and quantitative analyses will be used to evaluate the intervention from the perspectives of the women receiving the intervention, facilitators, site investigators and project management staff. The evaluation will use evaluation datasets, administratively collected process data accessed during monitoring visits, check lists and logs, quantitative participant evaluation surveys, semistructured interviews and focus group discussions. Interview participants will be recruited using maximum variation purposive sampling. We will undertake thematic analysis of all qualitative data, conducted contemporaneously with data collection until thematic saturation has been achieved. To triangulate data, the analysis team will engage in constant iterative comparison among data from various stakeholders. Ethics and dissemination Ethics approval has been obtained from the respective human research ethics committees of the All India Institute of Medical Sciences, New Delhi, India; University of Sydney, New South

\section{Strengths and limitations of this study}

- This study will be one of the first process evaluations of an adequately powered trial of a pragmatic lifestyle intervention for type 2 diabetes mellitus/ pre-diabetes prevention in women with gestational diabetes mellitus (GDM) within resource-constrained environments.

- The evaluation will be conducted from the perspectives of women participants, facilitators, site investigators and project management staff.

- The study design has been informed by the HeLP-her and PregDiabCare Programmes which established the acceptability and feasibility of low-intensity lifestyle interventions for women with GDM.

- Two highly complementary theories, Michie's Behaviour Change Theory and Normalisation Process Theory were used to design the qualitative tools.

- A potential limitation of large-scale trials is that some of the nuance pertaining to site-specific context can be lost during analysis. This will be address by including local researchers from a range of sites across the three study countries.

Wales, Australia; and site-specific approval at each local site in the three countries: India, Bangladesh and Sri Lanka. This includes approvals from the Institutional Ethics Committee at King Edwards Memorial Hospital, Maharaja Agrasen Hospital, Centre for Disease Control New Delhi, Goa Medical College, Jawaharlal Institute of Postgraduate Medical Education and Research, Madras Diabetes Research Foundation, Christian Medical College Vellore, Fernandez Hospital Foundation, Castle Street Hospital for Women, University of Kelaniya, Topiwala National Medical College and BYL Nair Charitable Hospital, Birdem General Hospital and the International Centre for Diarrhoeal Disease Research. Findings will be documented in academic publications, presentations at scientific meetings and stakeholder workshops. 
Trial registration numbers Clinical Trials Registry of India (CTRI/2017/06/008744); Sri Lanka Clinical Trials Registry (SLCTR/2017/001) and ClinicalTrials.gov Registry (NCT03305939); Preresults.

\section{INTRODUCTION}

In India, up to $25 \%$ of the 25 million pregnancies occurring each year are associated with gestational diabetes mellitus (GDM) and this proportion is rapidly increasing. ${ }^{1-3}$ Prevalence estimates using the International Association of Diabetes and Pregnancy Study Groups in Sri Lanka (23\%) are similar and in Bangladesh $(10 \%)$ are somewhat lower. ${ }^{4}$ Prior GDM is an established risk factor for the future development of type 2 diabetes mellitus (T2DM). ${ }^{6}$ Systematic review evidence, using international and specified local criteria, indicates a sevenfold greater risk for T2DM conversion in women with GDM compared with normoglycaemic pregnancies. ${ }^{7}$ Further, $35 \%-60 \%$ of Indian women with GDM will develop T2DM or pre-diabetes within 5 years of childbirth, more than double the rate observed in largely White populations. ${ }^{8-11}$ Onset of T2DM is preventable or can be delayed through sustained changes to diet, exercise and self-care (proactively engaging in activities to preserve and improve health). Yet, to date, there have been no completed adequately powered trials of interventions to demonstrate such an effect in South Asian women.

The Lifestyle InterVention IN Gestational diabetes (LIVING) Study is a randomised controlled trial (RCT) of a low-intensity lifestyle modification programme tailored to women with GDM in India, Bangladesh and Sri Lanka. The multiple interacting components of this complex intervention were informed by several successful studies in the prevention of T2DM and weight gain, which have been described elsewhere. ${ }^{11-14}$ The multicentre study is being undertaken over 60 months (December 2015 to December 2020). ${ }^{15}$ A total of 1414 women with prior GDM will be recruited from a total of up to 24 hospitals in these three countries. Women are recruited into the study in pregnancy to assess for GDM, and 3-18 months post-childbirth, if diagnosed with GDM in the index pregnancy, to assess glycaemic status for eligibility to participate in the trial. Women eligible for participation in the trial are therefore randomised to the control or intervention group 3-18 months after childbirth. Women are excluded from the trial if travel time to hospital is greater than 2 hours; a household mobile telephone is not available; if steroids are used during pregnancy or if they are likely to move residence in the next 3 years. Follow-up visits are conducted for all participants every 6 months from the data of randomisation. The intervention is designed to take 48-52 weeks from randomisation to have all of its components delivered. Further detail on the inclusion and exclusion criteria, the calculation of the number of subjects and how they will be recruited is in the main trial protocol. ${ }^{15}$ The primary outcome of the randomised trial is the proportion of women with a change of glycaemic category at or prior to the final visit: (1) normal glucose tolerance to impaired fasting glucose (IFG) or impaired glucose tolerance (IGT) or T2DM; and (2) IFG or IGT to T2DM. The secondary outcomes of the study include change in body weight, waist circumference, blood pressure, fasting blood glucose, physical activity level and diet. ${ }^{15}$ The main trial outcomes will not be analysed in the process evaluation. However, we will present figures related to the study processes, for example, duration between childbirth and glycaemic status assessment, duration of the intervention and intervals between follow-up visits.

The intervention comprises of 4 face-to-face group sessions, 84 automated text or pre-recorded voice messages, a participant manual, monthly phone calls and 2 'intensification' sessions in the event that weight goals are not achieved within 6 months of the commencement of the intervention. The LIVING Study process evaluation conducted alongside the RCT will inform policy and decision makers about the reasons why, and the mechanisms through which, the intervention worked and whether the intervention is likely to be generalisable and scalable in real-world contexts.

An essential component of the intervention involves 'task-shifting', a strategy involving the redistribution of tasks among health workforce teams described elsewhere. ${ }^{16}$ Our implementation strategy reflects a withinsystem task-shifting approach, whereby the intervention is delivered by auxiliary nurse midwives or their equivalent (hereafter called 'facilitators') in each participating hospital. Facilitators will have undergone training and will be equipped with educational materials (in local languages), including a facilitator manual with a session guide for each group session.

\section{Aims and objectives}

The overall aim of this process evaluation is to evaluate and understand what worked, and why, during the implementation of the LIVING intervention. In addition, this study aims to provide additional information that will assist in the interpretation of the LIVING Study results. This study will address this aim by:

- Evaluating for whom the intervention was effective, and why.

- Evaluating whether the intervention was implemented appropriately, and as planned.

- Evaluating whether the intervention is generalisable, scalable and sustainable in a real-world setting.

\section{METHODS AND ANALYSIS}

\section{Patient and public involvement}

Patients were involved in the design and conduct of this research. The LIVING intervention builds on the prior HeLP-her and PregDiabCare Programmes. ${ }^{17} 18$ HeLP-her is a low-intensity lifestyle programme designed to prevent persistent postpartum weight gain among ethnically diverse Australian women. Key features of this programme include non-prescriptive simple health messages, small 
changes to behaviour, low participant burden and intervention delivery, including a mix of group sessions, phone and short message service text reminders. The intervention was associated with a significant weight difference $(>1 \mathrm{~kg})$ between intervention and control groups, favouring the former. PregDiabCare comprised of only six group sessions focused on diet and physical activity, with implementation in two Indian hospitals providing with preliminary evidence of feasibility and effectiveness. Building on these prior interventions, the LIVING Programme was developed through a process of formative research. The formative research was conducted in two hospitals in each of North and South India, Bangladesh and one from Sri Lanka, and included document analysis, process mapping, semistructured interviews and focus group discussions with all key stakeholders (healthcare providers, patients and advisers to policymakers). We used these data from this formative research and the theoretical models described above to identify which barriers and enablers needed to be addressed, as well as what modifications and combinations of HeLP-her and PregDiabCare would be most likely to target such barriers and facilitators.

The UK Medical Research Council (MRC) provides guidance on implementing and evaluating complex interventions. The guidelines advocate for the use of theory in order to explain specific elements of the intervention and the avenues through which it may work. Contributors to MRC guidance contend that this is vital to building an evidence base that informs policy and practice. ${ }^{19} 20$

\section{Methodological approaches informing our evaluation framework}

The Reach Effectiveness Adoption Implementation Maintenance (RE-AIM) framework will be used to inform the LIVING Study's process evaluation framework. ${ }^{21}$

The RE-AIM framework consists of five dimensions of health-related behaviour interventions: reach to the target population, effectiveness of the intervention, adoption by the intended recipients of the intervention, staff and institutions such as hospitals, implementation (including consistency of implementation and costs) and maintenance of the intervention effects in specific settings over time. ${ }^{19}$ These five dimensions aim to encourage researchers, evaluators and policymakers to identify the elements of health interventions that improve adoption, implementation and sustainability. The RE-AIM framework was considered highly suitable as the LIVING Programme was designed with the intention of broader adoption and implementation into policy and practice in the South Asian context, and has been used to design our evaluation framework (figure 1).

\section{Theories informing our research tools}

Two highly complementary theories, Michie's Behaviour Change Theory and Normalisation Process Theory (NPT) (table 1), are used to guide the design of our qualitative tools (eg, semistructured discussion schedules) within the overall evaluation framework (figure 1).22 23

The LIVING intervention has been designed to build the capacity and motivation of women to make small, achievable changes to their lifestyle. Thus, we required a theory that would take a complex, multilayered approach

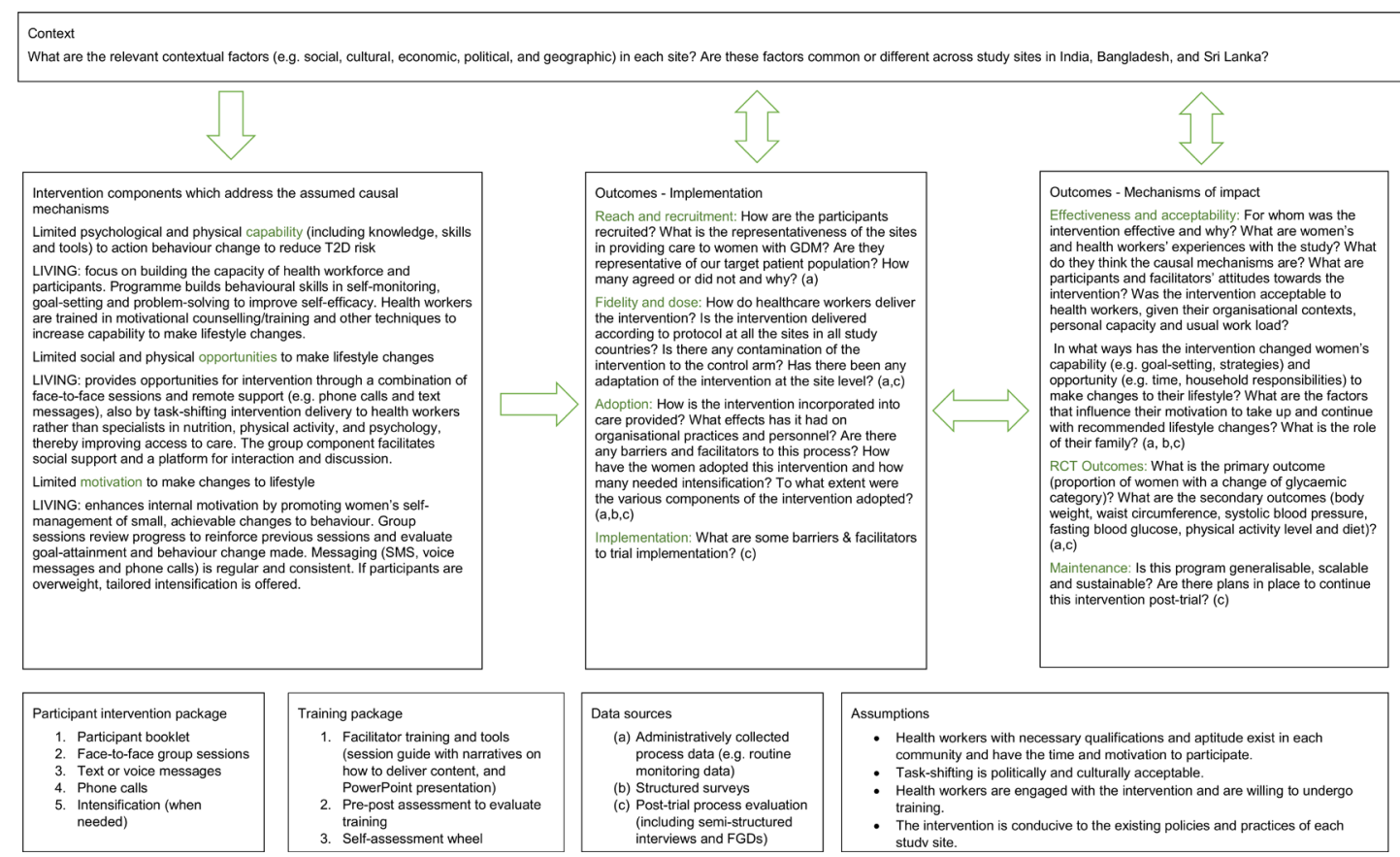

Figure 1 Process Evaluation Framework for the LIVING intervention to prevent or delay or delay onset of type 2 diabetes mellitus (T2DM) in women with gestational diabetes mellitus (GDM). FGDs, focus group discussions; LIVING, Lifestyle InterVention IN Gestational diabetes; RCT, randomised controlled trial; SMS, short message service. 
Table 1 Data collection tools and frameworks used to address process evaluation objectives

\begin{tabular}{lll}
\hline Objective & Data sources & Framework/s \\
\hline $\begin{array}{l}\text { Evaluating whether, and how, the intervention had } \\
\text { impact, for whom, and why }\end{array}$ & Surveys & Michie's Behaviour Change \\
& - Focusistructured interviews & Theory \\
& RE-AIM \\
Evaluating whether the trial was implemented & Routine monitoring data & RE-AIM \\
appropriately and as planned & Semistructured interviews & Normalisation Process Theory \\
Evaluating whether the results of the study are & Semistructured interviews & RE-AIM \\
generalisable, scalable and sustainable & Focus group discussions &
\end{tabular}

RE-AIM, Reach Effectiveness Adoption Implementation Maintenance.

to understanding individuals' behaviour change, and ideally, leverage the strengths and address the limitations of a plethora of other behaviour change frameworks. ${ }^{22}$ Michie's Theory is ideal in this regard as it consolidates key behaviour change frameworks, and includes a Capability Opportunity Motivation-Behaviour (COM-B) Model. Capability investigates individuals' physical and psychological capability. This includes knowledge, skills and tools. Opportunity refers to the social and physical opportunities available to the individual to perform a behaviour. Motivation refers to the level of motivation (as well as unconscious habits, and conscious thoughts and goals) that influence behaviour in reflective and automatic ways. The behaviour component looks at the type of behaviour sought, which is shaped by capability, opportunity and motivation. These sources of behaviour are positioned within a wheel, with the outer layers acknowledging the influence of intervention functions (eg, enablement, training, persuasion and education) and policy categories (eg, guidelines, fiscal measures and legislation).

However, the limitation of Michie's Behaviour Change Theory is that it is best suited to individual behaviour change, rather than organisational change. The third objective of this process evaluation is to evaluate whether the intervention is scalable and sustainable within a realworld setting. Thus, we required a conceptual framework to understand how the LIVING intervention's withinsystem task-shifting and new ways of organising and delivering healthcare would be adopted, implemented and practised. NPT is ideal as it is targeted at understanding the collective action and organisational behaviour required to introduce complex interventions to healthcare settings. The theory uses four constructs representing the work involved in establishing a new practice: coherence, cognitive participation, collective action and reflexive monitoring. Coherence refers to the sense-making work people undertake as individuals and collectively in order to operationalise new practices. Cognitive participation is the relational work done to sustain a community of practice from introducing a new practice (called the initiation phase) to defining the actions and procedures required to sustain a practice (called activation). Collective action refers to the operational work done to enact a set of practices (eg, considering workability and context). Reflexive monitoring is the appraisal work people do to assess and understand how new practices affect them and others around them. ${ }^{23}$ NPT will be used to inform the semistructured interviews with service providers (eg, health workers and management staff).

Together, the two theoretical frameworks will allow the research team to study the specific causal mechanisms through which the intervention seeks to make change, as well as the extent to which it is effective and acceptable, adopted by healthcare organisations, implemented as intended, and has the capacity to be scaled up and sustained (see figure 1 for evaluation framework).

\section{The LIVING Study process evaluation framework}

The evaluation framework (figure 1) demonstrates each of the intervention components and the relationships among them. First, the framework incorporates the intervention components addressing assumed causal mechanisms. These causal mechanisms, explained in detail in our logic model (figure 2), are intended to make it easier to understand why the intervention will work, and to facilitate external scrutiny of their plausibility. These assumptions are underpinned by the key elements of Michie's Behaviour Change Theory and COM-B model (capability, opportunity and motivation). Thus, it also serves to test the applicability and robustness of the theory in the context of lifestyle change in women with prior GDM in India, Bangladesh and Sri Lanka. Second, the framework incorporates implementation factors (eg, reach and recruitment, fidelity and dose, as well as adoption and implementation), mechanisms of impact (eg, effectiveness, acceptability and maintenance) and finally, the outcomes of the trial (to be assessed at the end of the study).

A mixed-methods design will be used to investigate the components of our process evaluation framework. This will involve the integration of both qualitative and quantitative data in the research process including evaluation datasets, administratively collected process data accessed during monitoring visits, check lists and logs filled at intervention sites, audit reports, quantitative participant 


\begin{tabular}{|c|c|c|c|c|c|}
\hline $\begin{array}{l}\text { LIVING } \\
\text { Intervention inputs }\end{array}$ & $\begin{array}{l}\text { Intervention processes } \\
\text { and actions }\end{array}$ & $\begin{array}{l}\text { Changes to capability, } \\
\text { motivation and } \\
\text { opportunity }\end{array}$ & $\begin{array}{l}\text { Changes at } \\
\text { organisational } \\
\text { level }\end{array}$ & $\begin{array}{l}\text { Immediate impacts } \\
\text { in women with GDM }\end{array}$ & $\begin{array}{l}\text { Health outcomes in } \\
\text { women with GDM }\end{array}$ \\
\hline \multirow[t]{2}{*}{ Participant manual } & \multirow{2}{*}{$\begin{array}{l}\text { Participants receive } \\
\text { information regarding the } \\
\text { health harms of GDM and } \\
\text { the potential of lifestyle } \\
\text { changes to prevent or } \\
\text { delay onset of T2DM }\end{array}$} & \multirow{2}{*}{$\begin{array}{l}\text { Increased knowledge } \\
\text { of health } \\
\text { consequences } \\
\text { triggers change to } \\
\text { diet, exercise, and }\end{array}$} & \multirow{4}{*}{$\begin{array}{l}\text { Greater } \\
\text { integration of } \\
\text { departments } \\
\text { (obstetrics and } \\
\text { gynaecology; } \\
\text { paediatrics; and } \\
\text { endocrinology } \\
\text { and diabetes) } \\
\text { and other support } \\
\text { staff (nursing, } \\
\text { dietetics, and } \\
\text { other disciplines) } \\
\text { for improved } \\
\text { continuity of care }\end{array}$} & $\begin{array}{l}\text { More women attend } \\
\text { postpartum } \\
\text { screening }\end{array}$ & $\begin{array}{l}\text { Prevention or delay } \\
\text { of onset of T2DM }\end{array}$ \\
\hline & & & & $\begin{array}{l}\text { More women with } \\
\text { GDM engage in }\end{array}$ & $\begin{array}{l}\text { Improved quality of } \\
\text { life and mental } \\
\text { health }\end{array}$ \\
\hline $\begin{array}{l}\text { Face-to-face group } \\
\text { sessions }\end{array}$ & \multirow{2}{*}{$\begin{array}{l}\text { Participants trained to } \\
\text { execute lifestyle change } \\
\text { through goal-setting, self- } \\
\text { management, and } \\
\text { problem-solving through } \\
\text { group sessions }\end{array}$} & $\begin{array}{l}\text { Participants set, } \\
\text { monitor and achieve } \\
\text { their health-related } \\
\text { goals }\end{array}$ & & $\begin{array}{l}\text { improve and } \\
\text { maintain good } \\
\text { mental health }\end{array}$ & \\
\hline $\begin{array}{l}\text { Text or voice } \\
\text { messages }\end{array}$ & & $\begin{array}{l}\text { Participants devise } \\
\text { strategies to improve }\end{array}$ & & \multirow{2}{*}{$\begin{array}{l}\text { More women with } \\
\text { GDM maintain a } \\
\text { healthy weight } \\
\text { through changes to } \\
\text { diet (e.g. increased } \\
\text { consumption of fruit, } \\
\text { vegetables, and } \\
\text { grains; reductions in } \\
\text { sugar and salt) }\end{array}$} & \\
\hline $\begin{array}{l}\text { Motivational } \\
\text { interviewing via } \\
\text { telephone }\end{array}$ & \multirow{2}{*}{$\begin{array}{l}\text { Intervention facilitators } \\
\text { reinforce new skills and } \\
\text { knowledge through phone } \\
\text { calls, text messages and } \\
\text { offer intensification after } \\
\text { personalised barriers } \\
\text { assessment } \\
\end{array}$} & $\begin{array}{l}\text { overcome physical, } \\
\text { social, and } \\
\text { psychological } \\
\text { challenges to lifestyle } \\
\text { change }\end{array}$ & $\begin{array}{l}\text { Improved access } \\
\text { to care for } \\
\text { women with } \\
\text { GDM due to } \\
\text { within-system }\end{array}$ & & \\
\hline \multirow{2}{*}{ Intensification } & & & task-shifting & \multirow{3}{*}{$\begin{array}{l}\text { More women with } \\
\text { GDM exercise at } \\
\text { least thirty minutes } \\
\text { every day }\end{array}$} & \\
\hline & \multirow{2}{*}{$\begin{array}{l}\text { Group and individual } \\
\text { sessions provide culturally } \\
\text { appropriate social support } \\
\text { for participants to } \\
\text { understand GDM and } \\
\text { make lifestyle changes }\end{array}$} & $\begin{array}{l}\text { Motivation to } \\
\text { maintain changes to }\end{array}$ & & & \\
\hline $\begin{array}{l}\text { Training of auxiliary } \\
\text { nurses to deliver } \\
\text { intervention }\end{array}$ & & $\begin{array}{l}\text { lifestyle is sustained } \\
\text { through text or voice } \\
\text { messages, and } \\
\text { phone calls }\end{array}$ & & & \\
\hline
\end{tabular}

evaluation surveys, semistructured interviews and focus group discussions.

1. Evaluation datasets: data from participants will be collected at randomisation and 6 monthly intervals thereafter, with oral glucose tolerance tests and HbA1c tests, alternately conducted at the follow-up 6 monthly visits, and both tests at the end-of-study visit. These assessments will include anthropometry, as well as surveys of physical activity and diet, using validated questionnaires.

2. Administrative data: data indicating eligibility and reasons for ineligibility will be used to assess generalisability of the intervention. Monitoring (at least one every 6 months) will be conducted by central study staff to ensure compliance with the protocol and any local requirements. During each visit, the study monitors will fill in a monitoring report on record maintenance, conduct of sessions and implementation of the programme, and offer feedback to ensure consistency of the implementation. The voice/text messaging report will indicate the number of successful messages sent every month as well as whether the voice message was heard completely or disconnected early.

3. Programme checklists and logbooks: programmespecific checklists were developed in conjunction with the research team records to assess implementation fidelity, recruitment, intervention delivery and programme context. Intervention session logs documenting attendance, collection of feedback and participation of group members will also be analysed.

4. Programme auditing: the delivery of the group sessions will be audited by direct observation, remote observation, or audio or video recording. These data will be analysed alongside the intervention session logs and checklists. All four sessions will be observed for the first group at each site, and one of any of the four sessions will be observed for all group sessions thereafter.

5. Quantitative participant evaluation survey: a barriers and facilitators evaluation survey (administered after the fourth (and final) group session, at approximately 6 months after randomisation) will be undertaken to explore what women have found to be enabling, and what challenging, in executing lifestyle modification following the group sessions. Surveys will list potential barriers to physical activity and healthy eating, as well as facilitators. Survey items are informed by the formative research as well as findings from previously conducted diabetes prevention programmes. Participants will also be able to mention barriers and enablers that are not listed in the evaluation form.

6. Semistructured interviews and group discussions: semistructured interviews and group discussions will be conducted with a subgroup of participants allocated to the intervention arm of the RCT, staff and stakeholders at the end of the study. Interviews will also be conducted using a maximum variation sampling technique to ensure that diverse opinions are gained from healthcare workers, hospital administrators and representatives of relevant government agencies with broad representation across the three countries.

7. Group session evaluation form: all women who attend group sessions will fill out a feedback form at the end of each session. They will score their overall experience of the intervention session including feedback on intervention content, its presentation, as well as the facilitator's skills and behaviour. 
In table 1 above, we describe how each of the data collection tools listed within the evaluation framework will be used.

\section{Evaluating whether and how the intervention had impact, for whom, and why \\ Surveys}

The barriers and facilitators evaluation survey (online supplemental appendix A) and group session evaluation form (online supplemental appendix B) will help us understand what aspects of women's lives including their living conditions, structural issues and level of support available are preventing them from achieving changes, and which aspects of the intervention they found useful, for example, self-motivation, scheduling, social support and information.

The group session evaluation form will explore whether the topics covered in the sessions were relevant to participants, whether they felt that the trainer was well prepared, whether the time allotted for the session was sufficient, and whether the content was organised and easy to follow.

\section{Evaluation datasets}

These data will be used to quantitatively evaluate effectiveness (eg, percent change in outcome variables at the end of the trial). These data will be used to compare characteristics of intensification and non-intensification participants to identify any trends in whom the intervention was most effective or least effective for.

\section{Semistructured interviews and focus group discussions}

Semistructured interviews (see online supplemental appendix C for interview schedule) and focus group discussions (see online supplemental appendix D for discussion schedule) will seek to gain a deeper understanding of the themes underpinning the surveys described above. From the interviews, we expect to understand participants' individual experiences of the intervention, while focus groups will be used to understand the similarities and differences in women's experiences. In addition, they will seek to address its gaps (eg, themes that participants raise in the surveys that were not listed by intervention developers in the preformulated survey tool). These data will contribute to a deeper understanding of what participants and the health workers delivering the intervention think the causal mechanisms are, as well as their general attitudes towards the intervention. The discussions will include a specific focus on how (from the perspectives of the participants and the health workers) the intervention impacts elements of Michie's Behaviour Change Theory, that is, women's capability, opportunity and motivation.

\section{Evaluating whether the trial was implemented appropriately and as planned}

In order to investigate the fidelity of the trial, that is, whether the components of the trial were delivered as intended and in appropriate detail, this process evaluation will use mixed methods. Routine monitoring data (eg, estimated number exposed to recruitment, percent screened for eligibility, percent ineligible for participation, actual number eligible and actual number who participated) and evaluation datasets will be used to assess reach (whether the intended audience came into contact with the intervention, and how). To assess implementation and whether poor implementation limited the effects of the intervention, we will use trial administration data including the actual number of phone calls, text messages received and sessions attended. The group session observation checklist (online supplemental appendix E), data obtained from programme auditing, as well as other administrative data collection tools mentioned above, will assess the management of group dynamics, the transmission and emphasis of key messages, the provision of all intervention materials and the participation of the group members.

In addition, pre-training and post-training assessment of facilitators will be undertaken to evaluate their training, and all facilitators will participate in a self-assessment wheel. We will use these data to understand how facilitators carried out their roles and responsibilities. These data will be supplemented with semistructured interviews and group discussions with participants and facilitators. The discussions will seek to understand, from various perspectives, issues of fidelity and dose (see online supplemental appendices $\mathrm{C}$ and $\mathrm{D}$ for discussion schedules).

\section{Evaluating whether the results of the study are generalisable,} scalable and sustainable

A third objective of this process evaluation will be to ascertain whether the intervention is generalisable and scalable. Our theoretical framework will allow researchers, evaluators and policymakers to pinpoint how the intervention, with reference to the key components of each theory incorporated into our framework, can be scaled up in India, Bangladesh and Sri Lanka, as well as tailored to other contexts. Focus groups and semistructured interviews will be used to understand the barriers and enablers to implementation. In addition, they will seek to understand how the complex intervention was embedded in organisational contexts as the result of individual and collective work to implement the programme.

We will investigate the extent to which the integration of the programme achieved coherence, cognitive participation, collective action and reflexive monitoring, as well as how it might be expected that this will continue in the future and in other contexts. Finally, using semistructured interviews with key stakeholders in decision-making and management, we will enquire about the extent to which current guidelines, environmental social planning, fiscal measures, and legislation, regulation and service provision are conducive to the scale-up and sustainability of the intervention at each site.

\section{Sampling}

Participants for the interviews and group discussions will be recruited using maximum variation purposive sampling. Of the 1414 women diagnosed with GDM recruited for 
the study, approximately half will receive the intervention. All data collection methods listed above, with the exception of the interviews and focus group discussions, will involve women in the intervention arm of the trial. For interviews and focus group discussions, 60-80 women with GDM (including those that were due to receive the intervention but did not attend any sessions) will be recruited at each of the sites in the North India, South India, Bangladesh and Sri Lanka regions to participate in the process evaluation. Evaluation data, session feedback and data from the barriers and facilitators survey will be obtained from each of the participants in the intervention. For the semistructured interviews, we will recruit 10 women from each of the four regions, arriving at a total of 40 participants. We will also recruit a facilitator, site investigator, project and hospital management staff, and policymaker from each of the four regions, totalling 20 interview participants. For the focus group discussions with participants, we will recruit five women from each of the four regions, arriving at a total of 20 participants. In total, we will recruit 60-80 interview and focus group discussion participants. We anticipate that this sample size will be sufficient to achieve thematic saturation based on previous GDM studies. In the event that thematic saturation has not been reached, we will increase our sample of participants. Participants will be recruited by the local site staff, and where relevant, reasons for not participating will be elicited. Written consent will be obtained for the interviews and group discussions.

\section{Analysis plan}

The quantitative data collected as part of this process evaluation will include checklists, logs and feedback surveys. Descriptive statistics will be generated for these data. The study outcome data, including change in glycaemic status, weight, waist circumference, diet and physical activity, will be analysed in the main trial. ${ }^{15}$

All interviews and group discussions will be audio recorded and transcribed. We will undertake thematic analysis of all qualitative data (moving from codes, to categories and finally themes). ${ }^{24}$ Data analysis will be conducted contemporaneously with data collection until thematic saturation has been achieved. The development of the coding framework will be informed by the RE-AIM framework as well as the two theories (Michie's Behaviour Change Theory and NPT) that have informed our interview and discussion schedules. The analysis team (JS, LKJ, DK and DP, and their research assistants) will consist of public health researchers with experience working in the South Asian region from nutrition, health and legal backgrounds. Members of the analysis team have been trained within institutions (The George Institute for Global Health and the All India Institute of Medical Sciences) with extensive experience in undertaking process evaluations alongside RCTs, developing and monitoring internal research practices that meet the highest standards of research integrity and ethics. The analysts will establish themes, under the headings of the evaluation framework: context, causal mechanisms, implementation, mechanisms of impact and outcomes. Given that the process evaluation is testing a hypothesised causal pathway (described in the logic model-figure 2), the analysis will aim to scrutinise the extent to which our logic model predicted the actual impact of the intervention, and also identify unexpected mechanisms.

To triangulate data, the analysis team will engage in constant iterative comparison among data from various stakeholders (eg, auxiliary nurse midwives, participants and management staff). Our analysis will identify components of the intervention, implementation and outcomes associated with a large degree of agreement, as well as identify any differences in experiences and perspectives among stakeholders. Data from other sources listed in the framework (including administratively collected process data and survey data) will be used to triangulate themes. Quantitative data will help inform fidelity and dose as described in the previous section. The framework will aid in triangulating qualitative and quantitative findings, as well as identifying the relationships among context, implementation, mechanisms of impact and outcomes. Data analyses will be ongoing, and process data will be analysed independently of the outcome evaluation data before the two sets of data are combined. Data will also be triangulated, to increase the reliability of results, using the multiple perspectives of analysts (based in India, Bangladesh, Sri Lanka and Australia) and the diverse disciplinary (social sciences, medical, legal), and cultural backgrounds of the analysts. In addition, at least one analyst (JS) has not been involved in the development of the intervention, and as such, we anticipate that this independent perspective will help to triangulate data and ensure the reliability of our results.

\section{Strengths and limitations}

A major strength of this study is that it will be one of the first process evaluations of an adequately powered trial of a pragmatic lifestyle intervention for T2DM/pre-diabetes in women with GDM within resource-constrained environments. In addition, the evaluation will be conducted from the perspectives of women participants, facilitators, site investigators and project management staff using two highly complementary theories, Michie's Behaviour Change Theory and NPT. A potential limitation of large scale trials is that some of the nuance pertaining to contextual factors (language, cultural norms and site specifics) may be lost during analysis and data management. These limitations will be address by involving local researchers from a variety of sites across the three study countries, during different periods of the study to enhance the reliability of the findings. An additional limitation is that women participating in the process evaluation will be recruited by facilitators at each study site, which may result in selection bias. In addition, an unintended consequence of our interview schedules being theory-informed may be that we miss factors (eg, other than capability, opportunity and motivation) impacting 
participant's ability to make changes to their lifestyle. We will address this by including open-ended questions.

\section{Ethics and dissemination}

All participants in discussions related to the process evaluation will be given information about the study, and their consent obtained. Ethics approval to conduct this research has been obtained from the University of Sydney, New South Wales, Australia. Additional site-specific ethics approval has been at each local site in the three countries: India, Bangladesh and Sri Lanka. This includes approvals from the Institutional Ethics Committee at King Edwards Memorial Hospital, Maharaja Agrasen Hospital, Centre for Disease Control New Delhi, Goa Medical College, Jawaharlal Institute of Postgraduate Medical Education and Research, Madras Diabetes Research Foundation, Christian Medical College Vellore, Fernandez Hospital Foundation, Castle Street Hospital for Women, University of Kelaniya, Topiwala National Medical College and BYL Nair Charitable Hospital, Birdem General Hospital and the International Centre for Diarrhoeal Disease Research.

In line with ethics requirements, data will be stored by The George Institute for Global Health on a storage platform (shared drive) only accessible by the research team. The audio recordings will be transcribed by a professional transcription service. The transcription service will sign a confidentiality agreement. To ensure the anonymity of participants, individual identifiers will be removed from the data. Direct identifiers (eg, name and address) and indirect (eg, date of birth, profession) will be removed during the transcription process. Data will be retained for a minimum of 7 years after completion of the project.

\section{Trial status}

The LIVING RCT was initiated in December 2015. Since 2015, the surveys, routine monitoring data and evaluation datasets described here have been routinely collected. Semistructured interviews and focus group discussions for the process evaluation will take place in 2020 and 2021. Follow-up will be completed by the end of 2020 . The establishment of the sites, intervention development and its site-specific contextualisation, as well as the staff training is complete. Results will be reported at the end of 2021 .

\section{Author affiliations \\ ${ }^{1}$ Faculty of Medicine, The George Institute for Global Health, University of New South Wales, Sydney, New South Wales, Australia \\ ${ }^{2}$ Department of Endocrinology, All India Institute of Medical Sciences, New Delhi, India \\ ${ }^{3}$ Division of Epidemiology, The George Institute for Global Health, Hyderabad, India ${ }^{4}$ Faculty of Medicine, University of New South Wales, Sydney, New South Wales, Australia \\ ${ }^{5}$ Prasanna School of Public Health, MAHE, Manipal, India \\ ${ }^{6}$ Monash Centre for Health Research and Implementation, Monash University, Melbourne, Victoria, Australia \\ ${ }^{7}$ Endocrine Unit, Department of Medicine, Goa Medical College, Goa, India}

Twitter Janani Shanthosh @JanChildRights and Devarsetty Praveen @drpraveend Contributors All authors contributed to the conceptualisation and design of this protocol. JS wrote the first draft and led the writing process. DK, LKJ and DP provided significant guidance on all aspects of the methodology and data collection instruments. AP, YG, NT, SJ, HJT, AD and RJ contributed to the refinement of the paper over numerous iterations.

Funding The study is funded by a National Health and Medical Research Council of Australia (NHMRC) Global Alliance for Chronic Diseases (GACD) Grant (APP1093171) and Indian Council of Medical Research (ICMR) GACD Grant.

Disclaimer The NHMRC and ICMR do not/will not have any role in the study design, management, data analysis and interpretation, writing of the report or the decision to submit the report for publication.

Competing interests None declared.

Patient consent for publication Not required.

Provenance and peer review Not commissioned; externally peer reviewed.

Supplemental material This content has been supplied by the author(s). It has not been vetted by BMJ Publishing Group Limited (BMJ) and may not have been peer-reviewed. Any opinions or recommendations discussed are solely those of the author(s) and are not endorsed by BMJ. BMJ disclaims all liability and responsibility arising from any reliance placed on the content. Where the content includes any translated material, BMJ does not warrant the accuracy and reliability of the translations (including but not limited to local regulations, clinical guidelines, terminology, drug names and drug dosages), and is not responsible for any error and/or omissions arising from translation and adaptation or otherwise.

Open access This is an open access article distributed in accordance with the Creative Commons Attribution Non Commercial (CC BY-NC 4.0) license, which permits others to distribute, remix, adapt, build upon this work non-commercially, and license their derivative works on different terms, provided the original work is properly cited, appropriate credit is given, any changes made indicated, and the use is non-commercial. See: http://creativecommons.org/licenses/by-nc/4.0/.

ORCID iDs

Janani Shanthosh http://orcid.org/0000-0002-1297-2400

Rohina Joshi http://orcid.org/0000-0002-3374-401X

\section{REFERENCES}

1 Ramachandran A, Snehalatha C, Shyamala P, et al. Prevalence of diabetes in pregnant women - a study from southern India. Diabetes Res Clin Pract 1994;25:71-4.

2 Grewal E, Kansara S, Kachhawa G, et al. Prediction of gestational diabetes mellitus at 24 to 28 weeks of gestation by using firsttrimester insulin sensitivity indices in Asian Indian subjects. Metabolism 2012;61:715-20.

3 Zargar AH, Sheikh MI, Bashir MI, et al. Prevalence of gestational diabetes mellitus in Kashmiri women from the Indian subcontinent. Diabetes Res Clin Pract 2004;66:139-45.

4 Guariguata L, Linnenkamp U, Beagley J, et al. Global estimates of the prevalence of hyperglycaemia in pregnancy. Diabetes Res Clin Pract 2014;103:176-85.

5 Herath HM, Weerarathna TP, Weerasinghe NP. Is risk factor-based screening good enough to detect gestational diabetes mellitus in high-risk pregnant women? A Sri Lankan experience. Int J Prev Med 2016;7.

6 Ratner RE, Christophi CA, Metzger BE, et al. Prevention of diabetes in women with a history of gestational diabetes: effects of metformin and lifestyle interventions. J Clin Endocrinol Metab 2008;93:4774-9.

7 Bellamy L, Casas J-P, Hingorani AD, et al. Type 2 diabetes mellitus after gestational diabetes: a systematic review and meta-analysis. The Lancet 2009;373:1773-9.

8 Kale SD, Yajnik CS, Kulkarni SR, et al. High risk of diabetes and metabolic syndrome in Indian women with gestational diabetes mellitus. Diabet Med 2004;21:1257-8.

9 Krishnaveni GV, Hill JC, Veena SR, et al. Gestational diabetes and the incidence of diabetes in the 5 years following the index pregnancy in South Indian women. Diabetes Res Clin Pract 2007;78:398-404.

10 Mahalakshmi MM, Bhavadharini B, Kumar M, et al. Clinical profile, outcomes, and progression to type 2 diabetes among Indian women with gestational diabetes mellitus seen at a diabetes center in South India. Indian J Endocrinol Metab 2014;18:400.

11 Gupta Y, Kapoor D, Desai A, et al. Conversion of gestational diabetes mellitus to future Type 2 diabetes mellitus and the predictive value of $\mathrm{HbA}_{1 \mathrm{c}}$ in an Indian cohort. Diabet Med 2017;34:37-43.

12 Knowler W, Barrett-Connor E, Fowler S, et al. Reduction in the incidence of type 2 diabetes with lifestyle intervention or metformin. Scand J Med Sci Sports 2003;13:208. 
13 Ramachandran A, Snehalatha C, Mary S, et al. The Indian diabetes prevention programme shows that lifestyle modification and metformin prevent type 2 diabetes in Asian Indian subjects with impaired glucose tolerance (IDPP-1). Diabetologia 2006;49:289-97.

14 Lombard C, Deeks A, Jolley D, et al. A low intensity, community based lifestyle programme to prevent weight gain in women with young children: cluster randomised controlled trial. BMJ 2010;341:c3215

15 Gupta Y, Kapoor D, Josyula LK, et al. A lifestyle intervention programme for the prevention of Type 2 diabetes mellitus among South Asian women with gestational diabetes mellitus [LIVING study]: protocol for a randomized trial. Diabet. Med. 2019;36:243-51.

16 Joshi R, Alim M, Kengne AP, et al. Task shifting for noncommunicable disease management in low and middle income countries - a systematic review. PLoS One 2014;9:e103754

17 Kapoor D, Gupta Y, Desai A, et al. Lifestyle intervention programme for Indian women with history of gestational diabetes mellitus. Glob. Health Epidemiol. 2019;4.

18 Kozica SL, Lombard CB, Harrison CL, et al. Evaluation of a large healthy lifestyle program: informing program implementation and scale-up in the prevention of obesity. Implementation Sci 2016;11:151.

19 De Silva MJ, Breuer E, Lee L, et al. Theory of Change: a theorydriven approach to enhance the Medical Research Council's framework for complex interventions. Trials 2014;15:267.

20 Craig P, Dieppe P, Macintyre S, et al. Developing and evaluating complex interventions: the new medical Research Council guidance. BMJ 2008;337:a1655.

21 Glasgow RE, Vogt TM, Boles SM. Evaluating the public health impact of health promotion interventions: the RE-AIM framework. Am J Public Health 1999;89:1322-7.

22 Michie S, van Stralen MM, West R. The behaviour change wheel: a new method for characterising and designing behaviour change interventions. Implementation Sci 2011;6:42.

23 May CR, Mair F, Finch T, et al. Development of a theory of implementation and integration: normalization process theory. Implementation Sci 2009;4:29.

24 Bradley EH, Curry LA, Devers KJ. Qualitative data analysis for health services research: developing taxonomy, themes, and theory. Health Serv Res 2007;42:1758-72. 